\title{
Réalisation \\ de l'émissaire de La Salie au sud d'Arcachon
}

PAR

\author{
J.-M. Barbier \\ Ingénieur des Ponts et Chaussées \\ Direction Départementale de l'Équipement \\ de la Gironde
}

Le Syndicat Intercommunal du Bassin d'Arcachon a réalisé, en 1973, pour le rejet en mer des eaux usées de toutes les communes riveraines du Bassin et des papeteries de la Cellulose du Pin à Facture, une estacade de $850 \mathrm{~m}$ de long au lieu-dit La Salie, au sud d'Arcachon, dans le golfe de Gascogne.

\section{Raccourci historique}

Vers 1950, le premier projet d'assainissement du Bassin d'Arcachon distinguait cinq groupes de communes, équipés chacun d'une station d'épuration avec stérilisation des eaux (pour protéger la conchyliculture) avant leur rejet dans le Bassin. Lorsqu'en 1963 les communes d'Arcachon, Gujan-Mestras, La Teste décidèrent la réalisation de ce projet, le principe du rejet d'effluents, même épurés, dans le Bassin d'Arcachon fut unanimement critiqué, comme n'offrant pas les garanties nécessaires de sécurité.

$1965:$ Un nouveau projet fut dressé (1965-1967) sur le principe de base qu'aucune eau usée, même épurée, ne doit être rejetée au Bassin; il comportait essentiellement :

- un collecteur général qui ceinture le Bassin d'Arcachon, capte les eaux usées des réseaux urbains traités en système séparatif et les eaux de la Cellulose du Pin;

- des stations de refoulement;

- un exutoire en mer.

Dans la zone de rejet, l'hydrographie, la courantologie, le comportement des effluents dans l'océan, en particulier leur dispersion et leur toxicité, ont fait l'objet d'études préalables menées par le Centre d'Études et de Recherches de Biologie et d'Océanographie Médicale (C.E.R.B.O.M.), et par le Laboratoire Central d'Hydraulique de France
(L.C.H.F.). Ces études conduisirent à fixer le point de rejet a $5,5 \mathrm{~km}$ au large de la plage de La Salie, à environ $15 \mathrm{~km}$ du Bassin d'Arcachon, ce avec l'hypothèse d'effluents non épurés et d'un débit de 2 à $3 \mathrm{~m}^{3} / \mathrm{s}$. 
Fin 1970: La première tranche fonctionnelle de travaux terrestres (commencée en mai 1968) était achevée: $30.5 \mathrm{~km}$ de canalisation de diamètre $1200 \mathrm{~mm}$ à $1500 \mathrm{~mm}$ (dont $3077 \mathrm{~m}$ de galeries), et 2 stations de refoulement permettaient le rejet des effluents des papeteries de la Cellulose du Pin. à lOcéan.

Mais la construction de l'émissaire en mer. confiée au début de 1970 à l'Entreprise Harmstorf, n’était pas terminée. et le déversement sur la plage provoquait des odeurs (mercaptans) et des mousses. d'où les réactions de l'opinion et de la presse (début 1971).

Pour lété 197/. Harmstorf posa. aux frais de la Cellulose du Pin, une canalisation provisoire de $400 \mathrm{~m}$ de long composée d'un simple tuyau en hostalen de $1200 \mathrm{~mm}$ de diamètre. lesté intérieurement, et amarré avec des chaînes et des corps morts : cet émissaire permit la suppression des nuisances pendant la saison estivale, mais disparut avec les tempêtes du mois d'octobre.

En février 1972, la Société Harmstorf avait posé au large le diffuseur de $250 \mathrm{~m}$ de long. ensouillé $1500 \mathrm{~m}$ de canalisation $O 1200 \mathrm{~mm}$ à $2 \mathrm{~m}$ de profondeur par des fonds de 30 a $25 \mathrm{~m}$. quand elle fit faillite. laissant sur place un matériel important et des travaux inachevés et inexploitables.

Le maitre d'ouvrage fit face à cette situation malheureuse :

- il réalisa, aux frais de la Cellulose du Pin. une seconde canalisation provisoire composée d'un tuyau acier (1) $800 \mathrm{~mm}$. fixé sur une estacade en bois. construite à lavancement avec des moyens terrestres, au-dela de la laisse de basse mer:

- il relança un concours pour la construction de lémissaire définitif.

\section{Concours de 1972}

Aux termes du devis programme. l'objet de ce concours était la construction d'un émissaire de $5.5 \mathrm{~km}$. réutilisant ou non les $1750 \mathrm{~m}$ de tuyau posés par Harmstorf. La tenue de la canalisation sur la plage et dans la zone de déferlement étant le problème le plus délicat. il était demandé aux concurrents densouiller la canalisation dans cette zone à $6 \mathrm{~m}$ sous le fond (comme l'avait prévu Harmstorf) ou de franchir cette zone avec une estacade et un tuyau aérien. Les fonds présentent en effet une grande mobilité due a leur nature très meuble (sable fin). et aux conditions de mer très dures auxquelles ils sont soumis, et des variations de $3 \mathrm{~m}$ ont été observées sur la plage.

Aucun concurrent ne proposa densouiller la canalisation. operation trop difficile en raison

- de limportance du mouvement des fonds et du transport littoral:

- de la mise en suspension continue du sable par les vagues:

- de la difficulté pour les bateaux de saventurer dans cette zone.
Les offres se classaient en deux catégories techniques :

- des sca-line classiques directement posés sur le fond (mis en place par tirage), de diamètre $900 \mathrm{~mm}$ à $1200 \mathrm{~mm}$;

- des estacades (appontements sur pieux) de 800 à $1200 \mathrm{~m}$ de longueur (franchissement de la zone de déferlement) prolongées vers le large par un sea-line posé sur le fond.

Les arguments en faveur de la première solution sea-line posé sur le fond étaient les suivants :

- moindre coût;

- procédé de construction assez classique;

- enfouissement naturel au fur et à mesure de la mise en suspension des fonds dans les tempêtes, garantissant la sécurité

En fait, le choix reposait sur cette dernière affirmation, ni confirmée, ni infirmée par l'expérience, donc assez douteuse.

\section{Choix de la solution estacade}

Dans la plupart des références citées par les concurrents :

- ou bien le sea-line avait un diamètre inférieur à $900 \mathrm{~mm}$ :

- ou bien les conditions de mer étaient moins dures que celles du golfe de Gascogne;

- ou bien les fonds étaient moins mobiles et moins variables:

- ou bien la conduite avait été ensouillée par le poseur (ce qui portait à croire que cette précaution n était pas inutile).

Pour éclairer ce choix difficile, qui résultait de la quasi impossibilité d'ensouiller la canalisation et des réticences à choisir une estacade coûteuse et trop "visible » dans le paysage, sur la proposition de son maitre d'œuvre, la Direction de l'Equipement, le Syndicat maître d'ouvrage confia une mission dexpertise à M. Sireyjol du B.C.E.O.M., pour le jugement du concours, et demanda des études complémentaires au L.C.H.F., portant sur l'examen des conditions de stabilité de l'émissaire, notamment :

- le relevé des fonds dans le couloir de l'émissaire, des plans de houle:

- lexamen théorique et l'estimation des efforts sur la conduite:

- une expérimentation sur modèle physique destiné à préciser le comportement global de la conduite sur le fond.

Ce modèle réduit en canal à houle ne pouvait représenter fidèlement l'ensemble des phénomènes en cause, mais il fournit des indications non évidentes :

- la possibilité de déplacement latéral de l'émissaire (phénomène d’ailleurs observé in situ sur la canalisation provisoire en 1971);

- surtout l'absence d'ensouillement propre d'une conduite posée sur un fond sableux (avec cependant la 


\section{ELLEVATION}
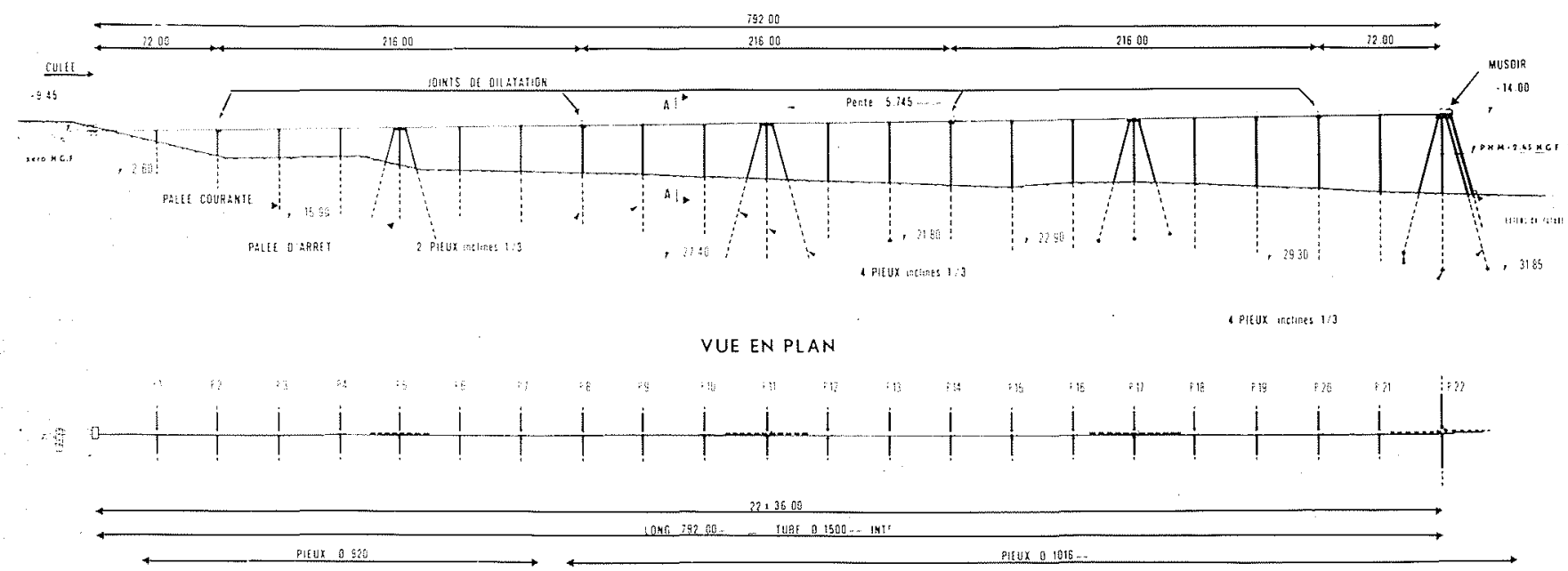

21

tendance à s"abaisser el à suivre les profils de plage les plus bas, et corrélativement les risques de désensouillement dus à un brusque abaissement du profil de la plage).

Ces deux phénomènes pouvaient entraîner la rupture de rémissaire. el contribuèrent à l'abandon de la solution sea-line.

Par ailleurs, le maitre d'ouvrage devait tenar compte :

- de contraintes financières, qui ne lui permettaient de construire que l'estacade en 1973:

- de l'évolution des doctrines d'assainissement. tendant à imposer l'épuration des effluents des agglomérations littorales avant leur rejet en mer (traduite par une décision du Ministre de l'Environnement C.I.A.N.E., décembre 1972) et qui pouvait permettre. avec une épuration des effluents, de réduire la longueur de l'émissaire.

Tels sont les éléments qui conduisirent le maître d'ouvrage à décider, en octobre 1972. la construction d'une estacade de $800 \mathrm{~m}$ de long pour le rejet en mer de ses eaux usées: les avantages de cette solution étaient en 1972 les suivants :

Elle permettait de:

- réaliser une partie d'émissaire absolument nécesscaire quel que soit plus tard le parti adopté pour le traitement des effluents et le prolongement de l'émissaire (le choix a été fait en 1973. d'épurer les effluents);

- franchir la zone la plus difficile. où la tenue d'un tuyau sur le fond de la mer présente des risques difficiles à éliminer:

- reporter, dès 1974, les déversements au-delà de la zone de déferlement permettant ainsi la disparition des nuisances (mousses) constatées sur la plage.

En outre, cette solution paraissait techniquement plus sûre:

- elle était évolutive ou progressive: le prolongement ultérieur par le séa-line était possible, mais pouvait être réétudié en fonction des observations réalisées in sillu;
- elle était relativement économique: les chantiers estacade et tirage du seca-line étant pratiquement indépendants l'un de l'autre, il était intéressant économiquement de différer le second chantier.

L’adjudicataire, l'Entreprise Gem-Hersent fut désignée en janvier 1973, et termina l'essentiel des travaux en un an.

\section{Description de l'ouvrage}

L'ouvrage, entièrement métallique, a une longueur totale de $792 \mathrm{~m}$ entre l'axe de la culée départ et l'axe de la palée terminale. Les travées de $36 \mathrm{~m}$ entre axes des appuis sont hyperstatiques.

A partir de la culée départ, il se compose de :

- 1 tronçon de $72 \mathrm{~m}$ :

- 3 tronçons de $216 \mathrm{~m}$;

- I tronçon de $72 \mathrm{~m}$.

La continuité de la conduite est assurée, entre deux tronçons sur palée, par des compensateurs doubles qui reprennent les dilatations de l'ouvrage. Il y a done 4 joints de dilatation et 2 joints d'extrémité.

La superstructure prend appui tous les $36 \mathrm{~m}$ sur des palées métalliques.

Les palées courantes sont composées de 2 tubes de $1 \mathrm{~m}$ de diamètre environ, réunis en tête par un chevêtre. Hls sont inclinés à 1 pour 3 dans le sens transversal pour reprendre les efforts dus au vent et à la composante transversale de la houle.

Les palées d'arêt n" 5,11 et 17 , implantées au centre de chaque élément, sont composées de quatre tubes de $1 \mathrm{~m}$ de diamètre environ. Ils sont inclinés à 1 pour 3 et placés dans deux plans perpendiculaires. Les tubes, orientés dans l'axe de l'ouvrage. s'opposent aux effets de la houle longitudinale, les tubes perpendiculaires à l'axe s'opposent aux 


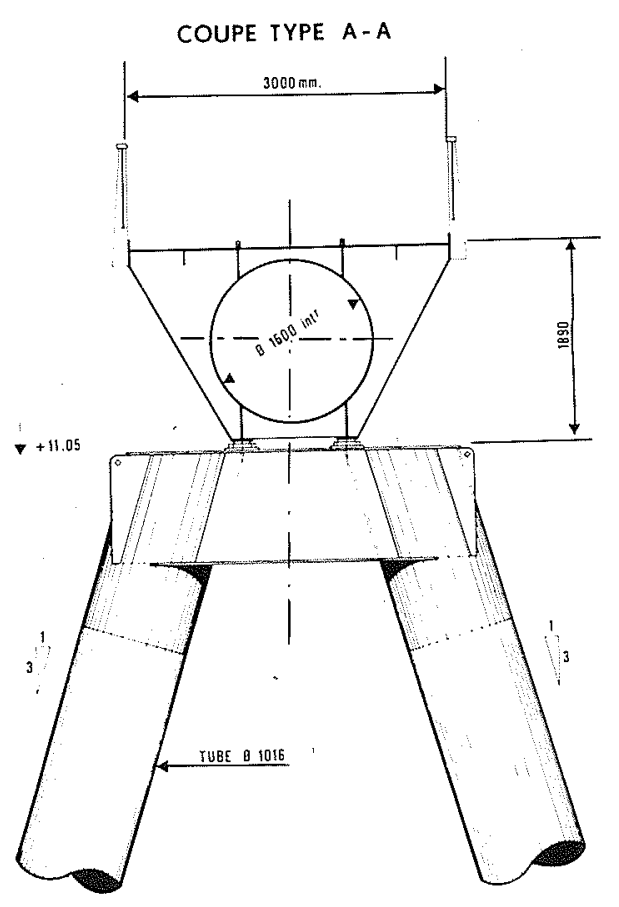

3 / Travée courante

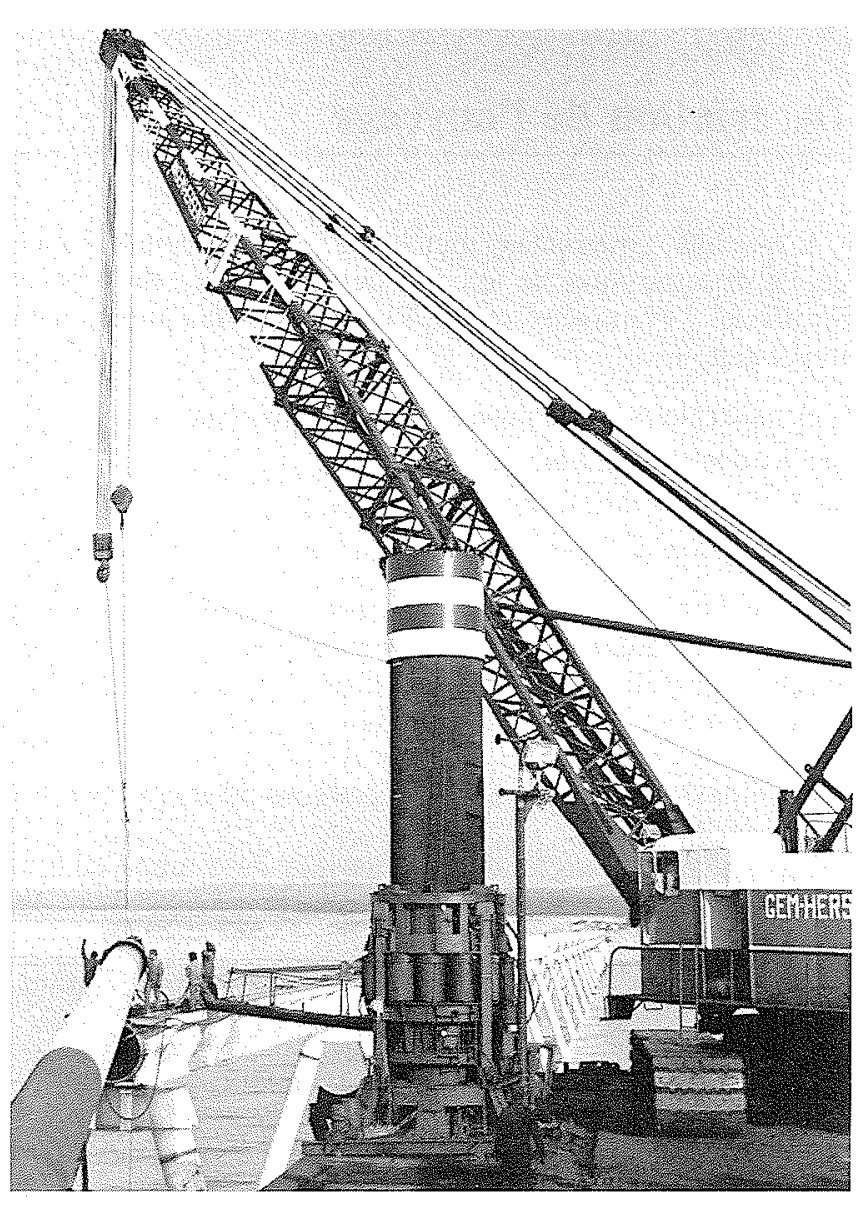

Photo A /
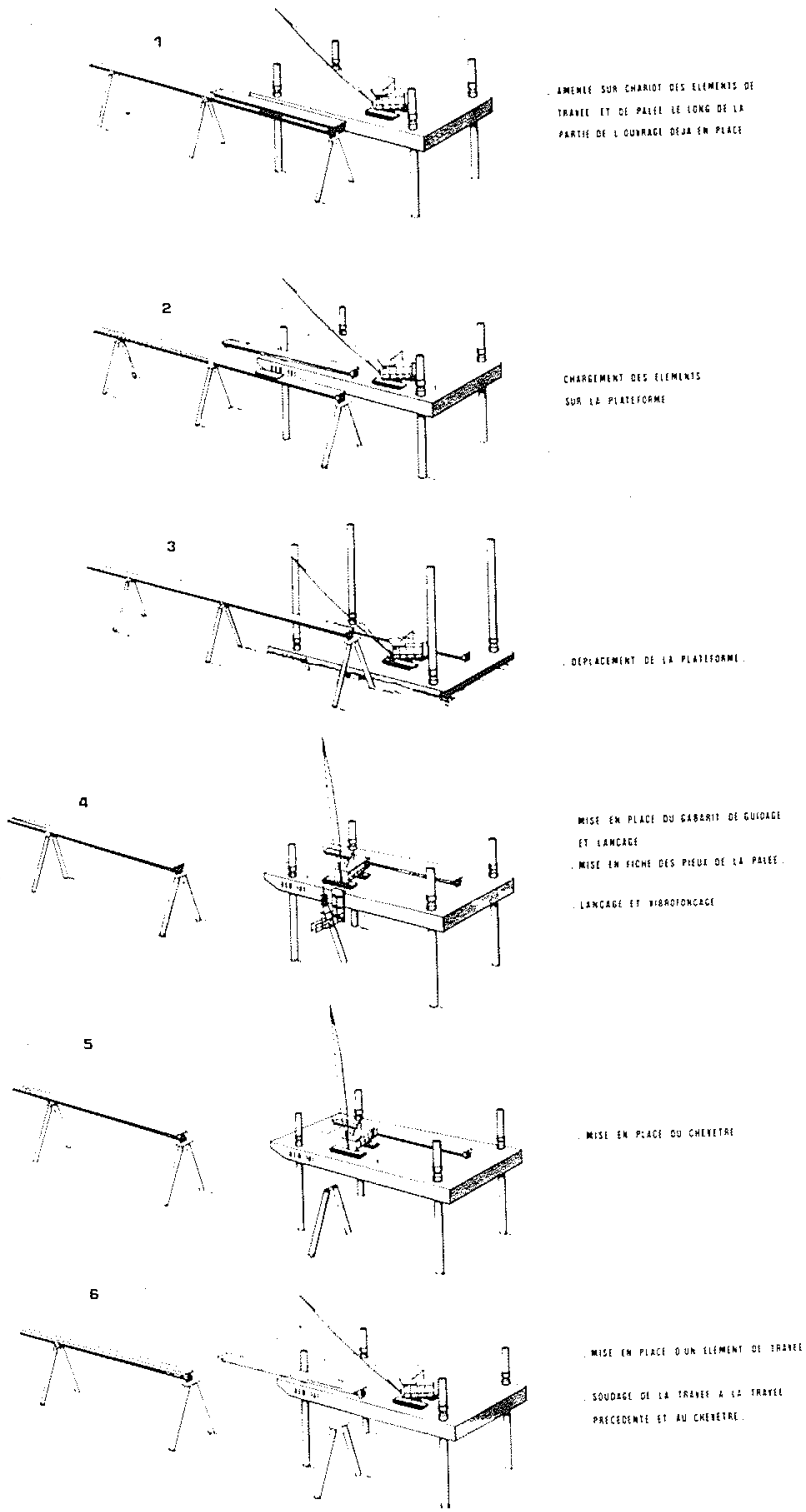

4 / Méthode de mise en place d'une travée courante 
efforts transversaux. Les quatre tubes sont réunis en tête par un chevêtre.

La palée terminale n" 22 est composée de quatre tubes métalliques de $1 \mathrm{~m}$ de diamètre inclinés à 1 pour 3 . Cette palée est complétée, côté large, par un tube supplémentaire de $1200 \mathrm{~mm}$ incliné à 1 pour 3 et orienté suivant l'axe de l'ouvrage. Il évacue les effluents par deux diaphragmes latéraux de $450 \mathrm{~mm}$ de diamètre. Dans les zones soumises à l'érosion, les pieux des palées sont protégés à leur pied par des viroles métalliques de $5,00 \mathrm{~m}$ de hauteur. de $10 \mathrm{~mm}$ d'épaisseur et concentriques au pieu.

La superstructure, entièrement métallique, est composée d'un tube de $1500 \mathrm{~mm}$ de diamètre. Ce tube est surmonté d'une plate-forme de circulation pour piétons de $3 \mathrm{~m}$ de large.

Le platelage de cette plate-forme, en tôle lisse, est solidarisé au tube par l'intermédiaire :

a) de deux longerons écartés de 1,00 m;

b) par des entretoises espacées tous les $4.00 \mathrm{~m}$.

Le platelage est raidi par des plats sur champ continu entre les entretoises. Au droit des deux longerons et sur le platelage, sont soudés deux carrés de $50 \times 40$ servant de rails pour lorries à voie métrique. A la partie inférieure deux longerons écartés de 1,00 sont solidaires du tube.

Le profil en long de l'ouvrage a été étudié de façon que la génératrice inférieure du tube de superstructure ne soit pas frappée par le crête de la houle. Cette génératrice se trouve à la cote +14 NGF (+ 16 hydro) à l'extrémité de l'ouvrage.

Un garde-corps de protection est fixé de chaque côté de la plate-forme de circulation.

Pour assurer la continuité, le tube est soudé sur le sommet des chevêtres des palées au moyen de diaphragmes. sauf au droit des compensateurs de dilatation, où une extrémité reste libre sur appui.

Les chevêtres, réunissant les pieux des palées en leur partie supérieure, sont constitués en caissons et réalisés par deux âmes espacées de 0,70 avec, à la partie supérieure, une semelle formant platine qui coiffe les têtes de pieux sur laquelle sont soudés les diaphragmes dans le prolongement des âmes. A la partie inférieure, une tôle horizontale qui relie les pieux forme semelle inférieure du caisson.

\section{Hypothèses de calcul prévues au marché}

Méthode de calcul conforme au fascicule 61 , titre $V$ du C.P.C. du Ministère de l'Equipement

- niveau maximum de la mer +6.00 hydro:

- courant transversal à l'ouvrage $2 \mathrm{~m} / \mathrm{s}$;

- inclinaison maximum de la houle sur l'ouvrage 35":

- affouillements (à prendre en compte pour le calcul des pieux et celui de la houle en chaque point) :

- $5 \mathrm{~m}$ de la palée 1 à la palée 6 .

- $3 \mathrm{~m}$ de la palée 7 à la palée 22;
- houle:

- période : 8 à $14 \mathrm{~s}$,

- amplitude : houle déferlante au point considéré;

— vent : $200 \mathrm{~kg} / \mathrm{m}^{2}$ de surface frappée;

- surcharges:

- effluent : $1.77 \mathrm{t} / \mathrm{ml}$ (sur toute la longueur),

- piétons : $375 \mathrm{~kg} / \mathrm{m}^{2}$ (moments en travée),

$323 \mathrm{Kg} / \mathrm{m}^{2}$ (moments sur appui)

- température $=27$ uniforme:

- sol de fondation assimilable à un sable $\varphi=30^{\prime \prime}$. $C=0$, densité immergée 1.1:

- aux épaisseurs dacier nécessaires pour les pieux telles qu'elles résultent de ces hypothèses ont été ajoutés $2 \mathrm{~mm}$ :

- aux fiches de pieux, calculées avec les hypothèses cidessus et les coefficients de sécurité habituels, a été ajoutée une surlongueur de $5 \mathrm{~m}$.

\section{CONSÉQUENCES DE CES HYPOTHESES POUR LA PALÉE D'EXTRÉMITÉ N" 22}

Composante horizontale de la houle sur I pieu : $50 \mathrm{t}$.

Effort de traction dans un pieu : $328 \mathrm{t}$.

Effort de compression dans un pieu 450 t. moment maximal $430 \mathrm{t} / \mathrm{m}$.

Fiche nécessaire (pour un pieu lisse) : $35 m+5 m=40 m$.

Longueur totale du pieu :

$\begin{aligned} & 40 \mathrm{~m} \\ & \text { fiche }\end{aligned}+\frac{14 \mathrm{~m}}{\text { hauteur NGF}}+\frac{9 \mathrm{~m}}{\text { profondeur }}=63 \mathrm{~m}$

\section{Mode de construction}

Les Chantiers Modernes ont réalisé les premiers travaux terrestres (accès au chantier, plate-forme pour les installations constructions de la culée...).

Les palées 1 à 4 ont été exécutées de la plage avec des moyens terrestres traditionnels: le fonçage des pieux était réalisé par un procédé mis en. œuvre par Soletanche (circulation inverse : une pompe Toyo descendue à l'intérieur du pieu désagrége le terrain et refoule l'eau chargée de sédiments par l'intérieur du pieu à l'extérieur): les derniers mètres sont vibrofoncés.

Les 22 travées métalliques de $36 \mathrm{~m}$ de long, pesant $40 \mathrm{t}$, ont été préfabriquées par C.F.E.M. dans son usine de Blanc-Misseron et acheminées par fer jusqu“à Cazaux. et par convoi exceptionnel routier sur le chantier.

Les palées 5 à 22 ont été mises en place par l'Entreprise Gem Hersent avec la plate-forme auto-élévatrice GEM 161, avec le concours de Soletanche pour le fonçage des pieux.

Les 7 premières travées de $36 \mathrm{~m}$ ont été lancées par I'Entreprise C.F.E.M. depuis la terre (avec un avant bec). Le procédé de construction au-delà de la palée 7 était le suivant

- sur la partie douvrage déjà réalisée. on acheminait les éléments de palée (pieux, chevêtres) et la travée jusquà la plate-forme où ils étaient chargés par une grue de 100 t Lima 2400 ; 


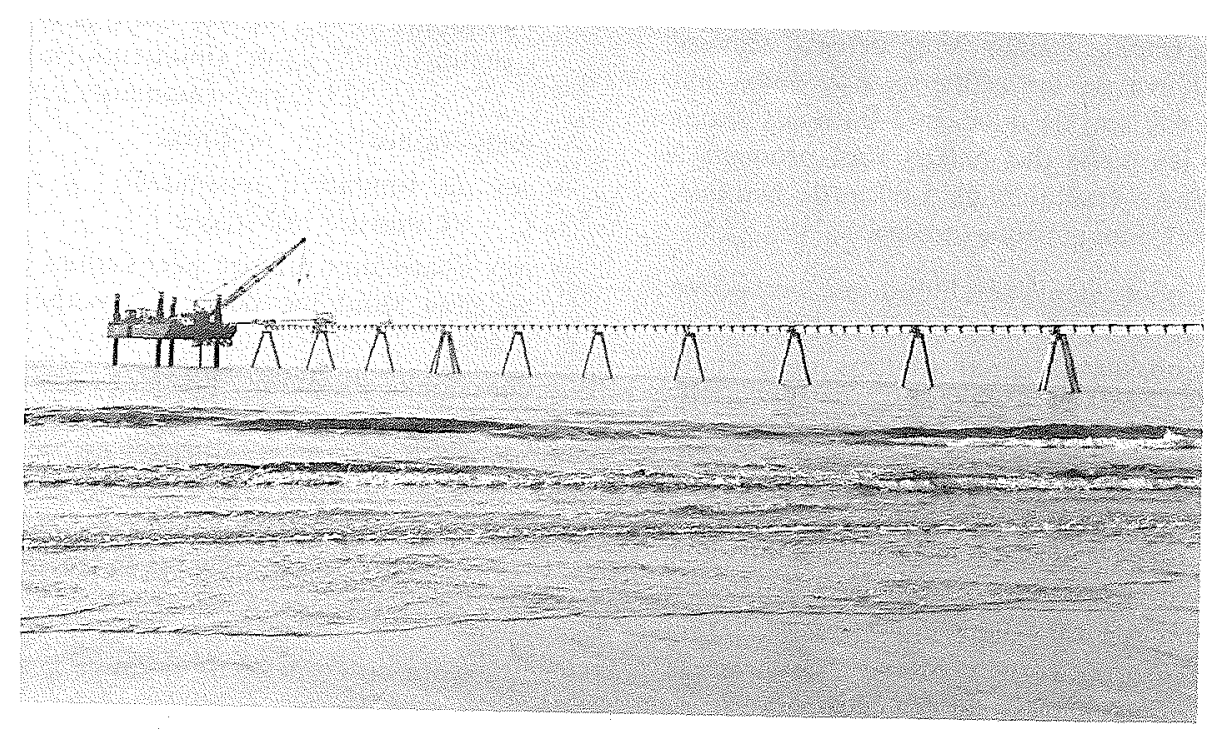

Photo B /

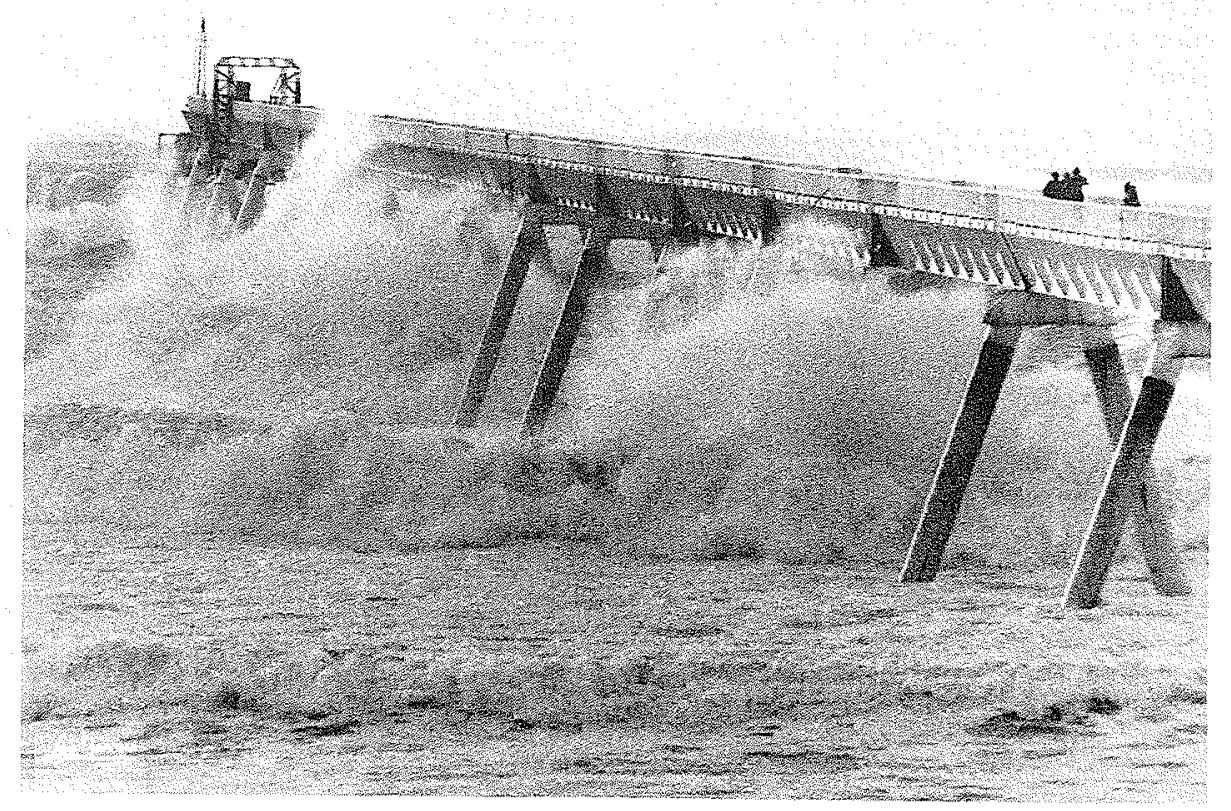

Photo C ! 
- la plate-forme auto-élévatrice se mettait en flottaison. se déplaçait sur ses ancres et s'auto-élevait au niveau de la palée suivante:

- le gabarit de guidage et lançage des pieux était réglé.

Les pieux étaient mis en fiche et remplis de sable. Le chevêtre était soudé après l'enlèvement du gabarit de guidage et la mise en place des viroles. La travée transportée par la barge était soudée sur la travée précédente et le chevêtre.

La barge pouvait être alors à nouveau alimentée avec les éléments du tronçon suivant, et le cycle dont la durée minimale était de trois jours, se répétait...

\section{Difficultés rencontrées} ter...

Les opérations sont plus faciles à décrire qưà exécu-

- La barge auto-élévatrice ne peut pas se déplacer par tout temps: les creux doivent rester inférieurs à $1 \mathrm{~m}$. le principal danger étant le talonnage de ses béquilles. Une fois auto-élevée, elle constitue une base vraiment stable et sûre qui permettait l'implantation des pieux avec le gabarit de guidage réglable à $20 \mathrm{~mm}$ près.

- Malgré la stabilité de cette plate-forme, le travail n'y était pas toujours possible, soit en cas de très forte houle. soit en cas de vent (la flèche de la grue. $50 \mathrm{~m}$ de long, devant alors rester couchée). la manutention et la soudure des travées ne pouvant se faire qu'en l'absence de vent.

- A ces difficultés dues au vent et à la houle s'ajoutait celle du terrain.

Le fonçage des pieux n'était pas facile et diverses techniques ou leur combinaison furent expérimentées, vibrofonçage, arrlift, circulation inverse, lançage proprement dit, la combinaison des deux dernières techniques (pompe désagrégatrice à l'intérieur du tube, lances soudées au tube à l'extérieur) donnait les meilleurs résultats.

La mise en place des pieux d'extrémité (fiche $40 \mathrm{~m}$ ) parut, dès le début du chantier, assez problématique. Comme cette longueur était due à la condition de non arrachement du pieu. l'entreprise réalisa des essais d'arrachement : un pieu lisse de $920 \mathrm{~mm}$ de diamètre. de $15 \mathrm{~m}$ de fiche, était arraché par un effort en tête de $72 \mathrm{t}$. Dans les mêmes conditions, un pieu muni en pied d'un sabot (ou d'un "harpon"), de $30 \mathrm{~cm}$ de haut et de $20 \mathrm{~cm}$ de large. n"a pu l'être avec une traction en tête de $340 \mathrm{t}$.
Cette solution. proposée par l'entrepreneur, de munir les pieux à leur pied d'une sorte de sabot fut donc adoptée et permit de réduire les fiches prévues d'environ moitié (pour le calcul, on considérait alors le pieu, non plus comme lisse. mais comme un pieu rugueux, en s'inspirant des études théoriques menées par M. Daniel Martin. Ingénieur TP à E.D.F.).

\section{Facteurs de succès}

Le succès du chantier provient de l'excellente préparation faite par l'Entreprise Hersent, de son expérience, de ses moyens (plate-forme auto-élévatrice), et évidemment des qualités des hommes qui ont conduit le chantier.

L'Entreprise a eu deux préoccupations qui méritent d'être soulignées:

1" Les opérations nautiques ont été réduites au strict minimum: elles étaient strictement limitées aux déplacements de la plate-forme auto-élévatrice; en dehors de cette manceuvre, pratiquement tout le chantier s'effectuait à partir de base fixe, stable, en dehors de la mer. La plateforme était reliée en permanence à la terre, ce qui est une sécurité pour le ravitaillement et le personnel (logé à terre). En dehors de l'amenée de la plate-forme, de la mise en place des ancres, aucun bateau n'était nécessaire sur le chantier.

$2^{4}$ Tous les travaux exécutés sur le site avaient été soigneusement étudiés et préparés pour être réalisés le plus rapidement possible. Ainsi la préfabrication était très poussée : travées et chevêtres étaient construits en usine, ceci afin de réduire au maximum le temps d'immobilisation de la barge par palée... (cette durée dépendait essentiellement du temps de fonçage des pieux). Des opérations telles que finition des soudures, pose des garde-corps, reprise des peintures étaient effectuées à partir d'un échafaudage mobile indépendant pour ne jamais retarder un déplacement de barge.

L’Entreprise fut désignée en janvier 1973, le marché fut signé fin mars 1973, en avril le chantier commençait.

La barge auto-élévatrice arrivait sur le site le 22 juin; elle repartait début décembre 1973, après la fin du gros auvre.

La mise en service de l'ouvrage eut lieu en mars 1974.

Le marché était entièrement forfaitaire, le coût de l'ouvrage s'élève (révision de prix incluse et TVA $17,6 \%$ incluse) à $19000000 \mathrm{~F}$. 


\section{Discussion}

Président : M. P. BergeroN

Je remercie vivement $M$. BARBIER, dit $M$. le Président, pour son exposé clair et remarquablement illustré par une belle série de diapositives; il me paraît particulièrement à sa place à la fin de cette séance car il met bien en lumière les difficultés rencontrées en pratique au passage de la zone de déferlement de la houle.

Le cas d'Arcachon est à cet égard typique, car la première tentative de pose d'une canalisation sur le fond - par les soins d'une entreprise allemande — s'est soldée par un échec; c'est ce qui a conduit à la solution de la canalisation sur estacade qui a été réalisée.

L'entreprise allemande, précise M. BARBLER, a commencé la pose de la conduite de diamètre $1100 \mathrm{~mm}$ par son extrémité en pleine mer à $5,5 \mathrm{~km}$ au large : elle a posé $1750 \mathrm{~m}$ de canalisation ensouillée à $2 \mathrm{~m}$ sous le fond de la mer par des profondeurs de $(-30 \mathrm{~m})$ lorsqu'elle a été déclarée en faillite, en janvier 1972, par suite de difficultés financières sans rapport avec son chantier d'Arcachon (notamment dévaluation du dollar).

A-t-on fait des mesures, depuis la mise en service de l'émissaire, pour vérifier l'efficacité du rejet au point de vue de la diffusion de l'effluent qui semblait effectivement être rabattu sur les plages avoisinantes, demande M. BIESEL (O.T.H.-L.C.H.F.)?

Oui, répond $M$. BARBIER, des expériences utilisant des colorants et la photographie du panache de diffusion ont été effectuées par le C.T.G.R.E.F. ainsi que des calculs théoriques d'interprétation des résultats ainsi obtenus.

Il faut noter que la décision initiale de placer le débouché de l'émissaire à $5,5 \mathrm{~km}$ au large de la côte avait été prise en vue d'éviter la pollution bactérienne des plages avoisinantes. Actuellement, la canalisation sur estacade ne transporte que les effluents de la papeterie de Facture de la Cellulose du Pin, à l'exclusion des effluents des réseaux urbains de la région. A partir du mois d'octobre 1975 , les premières amorces de ces réseaux urbains seront branchés, il est prévu la construction de stations d'épuration primaire.

Pour l'avenir, on peut envisager, soit un traitement plus poussé des effluents urbains (traitement tertiaire et éventullement chloration), soit un simple traitement primaire conjugué avec un allongement de l'émissaire pour amener son débouché à environ $5 \mathrm{~km}$ au large. Dans la situation actuelle, il n'est pas douteux que l'effluent peut revenir en partie sur la plage.

En réponse à une question de M. COLIANGE, M. BArbier donne les renseignements suivants :

Le diamètre des pieux de l'estacade est de $0.9 \mathrm{~m}$ à $1 \mathrm{~m}$. On avait prévu, contre la corrosion, une surépaisseur de $2 \mathrm{~mm}$ à la partie inférieure des pieux et une protection cathodique. Mais cette dernière n'a pas fonctionné correctement du fait qu'on n'a pas trouvé de prise de terre satisfaisante. Le fond de la mer est le siège d'une nappe d'eau douce très puissante et de très grande résistivité; il en résulte que toutes les prises de terre placées au fond marin) pour être encore en contact avec leau de mer plus ou moins diluée. Mais on cour le risque que ces électrodes soient au contact de l'eau de mer, seraient rapidement détériorées. On s'oriente vers des électrodes faiblement enterrées $(2$ à $3 \mathrm{~m}$ sous le fond marin) pour être encore en contact avec leau de mer plus ou moins diluée. Mais on court le risque que ces électrodes soient emportées avec le fond par grande tempête. Contre l'érosion mécanique, il a été prévu des viroles de renforcement des pieux dans la zone intéressée par le marnage; en fait, actuellement tous les pieux sont recouverts de moules et de coquillages et peut-être cette précaution était-elle inutile.

M. Couprie suggère aux organismes voués aux études océanographiques - tels que le C.N.E.X.O. - d'utiliser la longue estacade décrite par $M$. BARBIER pour installer une station de mesure des divers paramètres de la houle déferlante et, en particulier les long shore currents (déjà étudiés par les Américains) qu'elle engendre.

M. EzRATY signale qu'un projet similaire est en cours d'étude au nord de Bayonne pour l'étude des courants de houle; les mesures devraient pouvoir démarrer à la fin de 1975. Par ailleurs, grâce à l'estacade d'Arcachon, on pourrait, sous réserve de définir l'instrumentation adéquate, explorer la zone de déferlement sur $800 \mathrm{~m}$ environ; une telle étude serait certainement pleine d'enseignement.

En l'absence d'autre intervention, $M$. le Président clôt la discussion, et aussi la Session de juin du Comité Technique, en remerciant tous les conférenciers et toutes les personnes qui ont animé les débats. La séance est levée à $17 \mathrm{~h} 30$.

\section{Abstract \\ Construction of a sewage outfall at La Salie, near Arcachon (France)}

The sewage outfall at La Salie, near Arcachon (France), is the first pier of any size to be built on the Bay of Biscay. The pier is owned by S.I.A.C.R.I.B.A. ("Syndicat Intercommunal du Bassin d'Arcachon") and was engineered by the "Service de l'Equipement de la Gironde". Sea conditions are severe throughout the year and the entire length of the structure is often subjected to strong currents and breaking waves.

The offshore part of the $800 \mathrm{~m}$ long sewage outfall consist of a $1.50 \mathrm{~m}$ diameter overhead steel pipe. The pipe dis- charges effluent from a paper pulp plant and sewage from neighbouring towns. It is supported every $36 \mathrm{~m}$ on steel piles deeply embedded in the sandy sea bottom.

The project provides a good example of the use of jack-up barges, which offer a perfectly stable offshore working area and thereby enable construction work to be performed under severe conditions. The works were carried out by G.E.M. Hersent who subcontracted steel structure fabrication and pile driving to C.F.E.M. and Soletanche, respectively. 